\title{
Obtaining Nonlinear Frequency Responses from Broadband Testing
}

\author{
Etienne Gourc ${ }^{* 1}$, Chiara Grappasonni ${ }^{1}$, Jean-Philippe Noël ${ }^{1}$, Thibaut Detroux ${ }^{1}$, and \\ Gaëtan Kerschen ${ }^{1}$ \\ ${ }^{1}$ Space Structure and Systems Laboratory (S3L), Department of Aerospace and \\ Mechanical Engineering, University of Liège
}

\begin{abstract}
The objective of the paper is to obtain the frequency response curves of nonlinear mechanical systems from broadband testing. The proposed approach consists in coupling an identification method with a continuation method. Specifically, the frequency-domain nonlinear subspace identification (FNSI) method is first used to derive an experimental model of the structure in state space from broadband measurements. The harmonic balance method coupled with arclength continuation then utilizes this experimental model to compute the frequency response curves of the system. The method is demonstrated using a numerical example.
\end{abstract}

Keywords: subspace identification, continuation, harmonic balance, nonlinear, random excitation

\section{Introduction}

Nonlinear dynamical systems may exhibit complex behavior such as bifurcations, jump phenomenon or sensitivity to motion amplitude. When investigated using linear system identification method, these dynamical phenomena can be erroneously interpreted and lead to an inaccurate model.

Because nonlinearity is a frequent occurrence in engineering structures, there is a need for embedded methods that can be used to characterize the nonlinear behavior of a structure from experimental data $[8]$.

Among existing identification methods, subspace methods, developed for linear system identification $[13,10]$, are able to deal with multiple-input, multiple-output systems, were successfully applied for real-life applications [7]. Thanks to the feedback interpretation of the nonlinear structural dynamics [1], Marchesiello and Garibaldi proposed a time domain nonlinear subspace identification (TNSI) method able to estimate the frequency response function (FRF) of the underlying linear system as well as the nonlinear coefficients [9]. It has been shown that the method is sufficiently robust to identify appropriate nonlinear behavior from a set of basis functions. Later a frequency-domain version of the method, termed FNSI, was introduced [11]. Frequency-domain approaches are useful to reduce computational burden by selecting appropriate frequency bandwidth in the response spectrum for the identification. For greater flexibility allowing identification of complex nonlinearity, nonlinear basis function were replaced by cubic splines and the method was applied to the identification of bolted connections of a solar array panel [12].

The previous mentioned references aim at characterizing nonlinear systems using input-output data. The identified model is in the state-space form and the estimated matrices cannot be directly related to the system physical mass, damping and stiffness matrices. Therefore, as pointed out by Ewins et al., there is a need for a simulation tool to simulate directly the estimated model in the state-space frequency domain [6].

Different algorithms for the computation of periodic solutions and bifurcations of nonlinear structure can be found in the literature. Most of them rely on a continuation procedure to track periodic solutions along branches with respect to a control parameter (frequency of excitation or a system parameter) [2]. Among all the methods for computing periodic solutions in frequency domain, the

* etienne.gourc@ulg.ac.be 


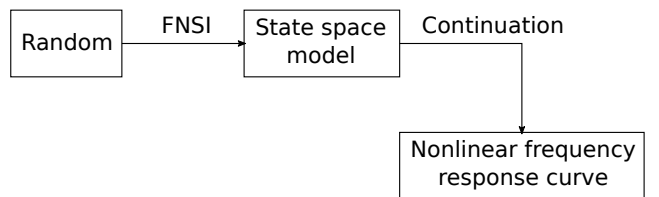

Figure 1: Diagram of the system identification methodology as addressed in the present paper.

harmonic balance (HB) is arguably the most used. The periodic signals are approximated by their Fourier coefficients, which become the new unknowns of the problem.

This method was applied to several industrial cases such as rotor-stator contact problems in turbomachinery [14], or to large-scale structures such as the nonlinear behavior of a full-scale vehicle [3]. Recently, the harmonic balance method has been used for the detection and tracking of codimension-1 bifurcations and applied to the analysis of the SmallSat spacecraft [5].

In this paper, an embedded approach coupling the FNSI method with a state-space harmonicbalance based continuation method is proposed and summarized in Fig. 1.

\section{Frequency-domain nonlinear subspace identification}

In this section, the frequency nonlinear subspace identification method (FNSI) is briefly recalled [11].

\subsection{Identification problem formulation}

The behavior of a mechanical system with discrete nonlinearities may be described by the following equation of motion

$$
M \ddot{q}(t)+C \dot{q}(t)+K q(t)+f(q(t), \dot{q}(t))=p(t)
$$

where $M, C, K \in \mathbb{R}^{r \times r}$ are the linear mass, viscous damping and stiffness matrices, $q(t), p(t) \in \mathbb{R}^{r}$ are the generalized displacement and external force vector. $f(t) \in \mathbb{R}^{r}$ is the nonlinear restoring force vector. The effect of the $s$ lumped nonlinearities is expressed as

$$
f(q(t), \dot{q}(t))=\sum_{j=1}^{s} \mu_{j} b_{j} g_{j}(q(t), \dot{q}(t))
$$

where $\mu_{j}$ are the nonlinear coefficients, $b_{j} \in \mathbb{R}^{r}$ is a Boolean vector indicating the location of the nonlinearity and $g_{j}(q(t), \dot{q}(t))$ is the nonlinear functional form. Defining the state vector $x=\left[q^{T}, \dot{q}^{T}\right]^{T} \in$ $\mathbb{R}^{n}(n=2 r)$, system (1) is rewritten in state space form as

$$
\begin{aligned}
\dot{x}(t) & =A_{c} x(t)+B_{c} e(p(t), q(t), \dot{q}(t)) \\
q(t) & =C x(t)+D e(p(t), q(t), \dot{q}(t))
\end{aligned}
$$

here, the subscript $c$ stands for continuous space. $e(t)=\left[p(t)^{T}, g_{1}(t), \ldots, g_{s}(t)\right]^{T} \in \mathbb{R}^{r+s}$ is the extended input vector which comes from the feedback interpretation of the nonlinear terms [11]. State space matrices $A_{c} \in \mathbb{R}^{n \times n}, B_{c} \in \mathbb{R}^{n \times(r+s)}, C \in \mathbb{R}^{r \times n}, D \in \mathbb{R}^{r \times(r+s)}$ are related to physical matrices as

$$
\begin{gathered}
A_{c}=\left[\begin{array}{cc}
0^{r \times r} & I^{r \times r} \\
-M^{-1} K & -M^{-1} C
\end{array}\right], \quad B_{c}=\left[\begin{array}{cccc}
0^{r \times r} & 0^{r \times 1} & \ldots & 0^{r \times 1} \\
M^{-1} & -\mu_{1} M^{-1} b_{1} & \ldots & -\mu_{s} M^{-1} b_{s}
\end{array}\right] \\
C=\left[\begin{array}{ll}
I^{r \times r} & 0^{r \times r}
\end{array}\right], \quad D=0^{r \times(r+s)}
\end{gathered}
$$

In order to reduce computational burden, the identification is performed in frequency domain. For improved numerical conditioning, a discrete-time translation is considered [9] before applying the discrete Fourier transform to system (3)

$$
\begin{aligned}
z_{k} X(k) & =A_{d} X(k)+B_{d} E(k) \\
Q(k) & =C X(k)+D E(k)
\end{aligned}
$$

where $z_{k}=\mathrm{e}^{2 j \pi k / N_{s}}, N_{s}$ is the number of recorded samples in the time series. Knowing the extended input $E$ and output $Q$, we want to determine the order of the system $n$ and the system matrices $A_{d}$, $B_{d}, C$ end $D$. 


\subsection{The outuput-state-input equation}

In practical applications, only a limited set of degree of freedom (DOF) in $p(t)$ and $q(t)$ are excited and observed, respectively. Therefore, the problem is preferably stated in terms of measured applied force $u(t) \in \mathbb{R}^{m \leq r}$ and displacement $y(t) \in \mathbb{R}^{l \leq r}$, so that $e(t) \in \mathbb{R}^{s+m}$. Equation (5) is rewritten as

$$
\begin{aligned}
z_{k} X(k) & =A_{d} X(k)+B_{d}^{e} E(k) \\
Y(k) & =C_{d} X(k)+D_{d}^{e} E(k)
\end{aligned}
$$

where $Y(k)$ is the discrete Fourier transform of $y(t)$ and the state space matrices are now a projection of the original matrices onto the observed and controlled DOFs. In what follows, the subscript $d$ is dropped for brevity. The measured input and output spectra are rearranged in block Hankel matrices

$$
Y_{i}=\left[\begin{array}{cccc}
Y(1) & Y(2) & \ldots & Y(N) \\
z_{1} Y(1) & z_{2} Y(2) & \ldots & z_{N} Y(N) \\
z_{1}^{2} Y(1) & z_{2}^{2} Y(2) & \ldots & z_{N}^{2} Y(N) \\
\vdots & \vdots & \ddots & \vdots \\
z_{1}^{i-1} Y(1) & z_{2}^{i-1} Y(2) & \ldots & z_{N}^{i-1} Y(N)
\end{array}\right] \in \mathbb{R}^{l i \times N}
$$

where $i$ is a user-defined index which must be chosen to encompass sufficient information to identify the system. $N$ is the number of non-necessary equidistant frequency lines taken for the identification procedure. The extended observability matrix $\Gamma_{i}$ and the lower block Toeplitz matrix $H_{i}$ are defined as

$$
\begin{aligned}
\Gamma_{i}= & {\left[\begin{array}{ccccc}
C^{T} & C A^{T} & C A^{2^{T}} & \ldots & C A^{i-1^{T}}
\end{array}\right]^{T} \in \mathbb{R}^{l i \times n} } \\
H_{i}= & {\left[\begin{array}{ccccc}
D^{e} & 0 & 0 & \ldots & 0 \\
C B^{e} & D^{e} & 0 & \ldots & 0 \\
C A B^{e} & C B^{e} & D^{e} & \ldots & 0 \\
\vdots & \vdots & \vdots & \ddots & \vdots \\
C A^{i-2} B^{e} & C A^{i-3} B^{e} & C A^{i-4} B^{e} & \ldots & D^{e}
\end{array}\right] \in \mathbb{R}^{l i \times(s+m) i} }
\end{aligned}
$$

By making recursive use of Eq. (6), the output-state-input matrix equation is obtained

$$
Y_{i}=\Gamma_{i} X+H_{i} E_{i}
$$

here $X \in \mathbb{R}^{n \times N}$ is the unknown state spectrum.

\subsection{Estimation of the state matrices and the order of the system}

The subspace identification method can now be applied to (9) to determine the order of the system and the matrices of the state space system (6). The algorithm consists of two main steps.

- First, the term depending on the input and the nonlinearities, namely $H_{i} E_{i}$ is eliminated. This task is achieved through an orthogonal projection onto the orthogonal complement of $E_{i}$. Then a singular value decomposition (SVD) of the result of the projection is performed. The order of the system and the estimated extended observability matrix are determined from the result of the SVD.

- The next step consist in computing the matrices of the state space system. Matrices $A$ and $C$ are easily computed by making use of the shift property of $\Gamma_{i}$. The procedure for computing matrices $B$ and $D$ is discussed in [11] (or in [13] for a general presentation of subspace-based identification methods).

Note that the state space matrices are obtained only within a similarity transformation matrix $T$, so that

$$
A_{c}=T \hat{A}_{c} T^{-1}, \quad B_{c}=T \hat{B}_{c}, \quad C=\hat{C} T^{-1}, \quad D=\hat{D}
$$

It is possible to define a similarity transformation matrix to express the identified matrices in the physical state-space domain (i.e. $x=\left[q^{T}, \dot{q}^{T}\right]^{T}$ ). Using Eq. (10), the problem is formulated as follows

$$
\begin{aligned}
& T \hat{A}_{c}=\left[\begin{array}{ll}
T_{1} & T_{2} \\
T_{3} & T_{4}
\end{array}\right]\left[\begin{array}{ll}
A_{1} & A_{2} \\
A_{3} & A_{4}
\end{array}\right]=\left[\begin{array}{cc}
0^{r \times r} & I^{r \times r} \\
-M^{-1} K & -M^{-1} C
\end{array}\right]\left[\begin{array}{ll}
T_{1} & T_{2} \\
T_{3} & T_{4}
\end{array}\right] \\
& \hat{C}=\left[\begin{array}{ll}
C_{1} & C_{2}
\end{array}\right]=\left[\begin{array}{ll}
I^{r \times r} & 0^{r \times r}
\end{array}\right]\left[\begin{array}{ll}
T_{1} & T_{2} \\
T_{3} & T_{4}
\end{array}\right]
\end{aligned}
$$


which gives

$$
\begin{aligned}
& T_{1} A_{1}+T_{2} A_{3}=T_{3} \\
& T_{1} A_{2}+T_{2} A_{4}=T_{4} \\
& C_{1}=T_{1} \\
& C_{2}=T_{2}
\end{aligned}
$$

Therefore the obtained similarity transformation matrix reads

$$
T=\left[\begin{array}{c}
\hat{C} \\
\hat{C} \hat{A}_{c}
\end{array}\right]
$$

Usually, a last procedure to compute the nonlinear coefficients $\mu_{j}$ from the estimated matrices is performed. However, this step is not necessary for the continuation procedure.

\section{Harmonic balance-based continuation method}

In this section, the computation of the periodic solution using the harmonic balance method is presented.

\subsection{Harmonic balance formulation}

The harmonic balance method is applied directly to the state-space system (3), where the state space matrices are those obtained using the FNSI method $\left(\hat{A}_{c}, \hat{B}_{c}, \hat{C}\right.$ and $\left.\hat{D}\right)$. In what follows, the hats are dropped for simplicity of notation. The state, output and input variables are approximated by Fourier series truncated to the $N$ - th harmonic

$$
\begin{aligned}
& x(t)=\frac{X_{0}}{\sqrt{2}}+\sum_{j=1}^{N} X_{c j} \cos \left(k_{j} \theta\right)+X_{s j} \sin \left(k_{j} \theta\right) \\
& q(t)=\frac{Q_{0}}{\sqrt{2}}+\sum_{j=1}^{N} Q_{c j} \cos \left(k_{j} \theta\right)+Q_{s j} \sin \left(k_{j} \theta\right) \\
& e(t)=\frac{E_{0}}{\sqrt{2}}+\sum_{j=1}^{N} E_{c j} \cos \left(k_{j} \theta\right)+E_{s j} \sin \left(k_{j} \theta\right)
\end{aligned}
$$

where $\theta=\omega t, \omega$ is the pulsation of excitation. $Q_{c j}$ and $Q_{s j}$ are the unknown Fourier coefficients related to the cosine and sine terms, respectively. Note that the Fourier coefficients of $e(t)$, depends on the Fourier coefficients of $q(t)$ due to the nonlinear basis functions $g_{j} . \quad k \in \mathbb{R}^{N}$ is the vector containing the different harmonics. The Fourier coefficients are gathered into vectors as follow

$$
\begin{aligned}
Q & =\left[\begin{array}{llllll}
Q_{0} & Q_{c 1} & Q_{s 1} & \ldots & Q_{c N} & Q_{s N}
\end{array}\right] \in \mathbb{R}^{(2 N+1) l} \\
X & =\left[\begin{array}{llllll}
X_{0} & X_{c 1} & X_{s 1} & \ldots & X_{c N} & X_{s N}
\end{array}\right] \in \mathbb{R}^{(2 N+1) n} \\
E & =\left[\begin{array}{llllll}
E_{0} & E_{c 1} & E_{s 1} & \ldots & E_{c N} & E_{s N}
\end{array}\right] \in \mathbb{R}^{(2 N+1)(s+m)}
\end{aligned}
$$

Using (15), the variables are rewritten in compact form as follow

$$
\begin{aligned}
& x(t)=\left(T(\theta) \otimes I_{n}\right) X \\
& q(t)=\left(T(\theta) \otimes I_{l}\right) Q \\
& e(t)=\left(T(\theta) \otimes I_{s+m}\right) E
\end{aligned}
$$

where $T(\theta)$ is a vector gathering the trigonometric functions as

$$
T(\theta)=\left[\begin{array}{llllll}
\frac{1}{\sqrt{2}} & \cos \left(k_{1} \theta\right) & \sin \left(k_{1} \theta\right) & \ldots & \cos \left(k_{N} \theta\right) & \sin \left(k_{N} \theta\right)
\end{array}\right] \in \mathbb{R}^{(2 N+1)}
$$

The time derivative of $x(t)$ can be written using a linear operator as

$$
\frac{d x}{d t}=\omega \frac{d}{d \theta}=\omega\left(\frac{d T(\theta)}{d \theta} \otimes I_{n}\right) X=\omega\left[(T(\theta) \nabla) \otimes I_{n}\right] X
$$

with

$$
\nabla=\left[\begin{array}{llll}
0 & & & \\
& \nabla_{1} & & \\
& & \ddots & \\
& & & \nabla_{N}
\end{array}\right] \quad \text { with } \nabla_{j}=\left[\begin{array}{cc}
0 & k_{j} \\
-k_{j} & 0
\end{array}\right]
$$

Substituting Eqs. $(16,18)$ into $(3)$ and applying Galerkin procedure gives 


$$
\begin{aligned}
\omega\left(\nabla \otimes I_{n}\right) X & =\left(I_{(2 N+1)} \otimes A_{c}\right) X+\left(I_{(2 N+1)} \otimes B_{c}\right) E \\
Q & =\left(I_{(2 N+1)} \otimes C\right) X+\left(I_{(2 N+1)} \otimes D\right) E
\end{aligned}
$$

Rearranging, the following residue equation is obtained

$$
h(Q, \omega) \equiv Q-G(\omega) E(Q)=0
$$

with

$$
\begin{gathered}
G(\omega)=\left(I_{(2 N+1)} \otimes C\right) \Lambda^{-1}\left(I_{(2 N+1)} \otimes B_{c}\right)+\left(I_{(2 N+1)} \otimes D\right) \\
\Lambda=\omega\left(\nabla \otimes I_{n}\right)-\left(I_{(2 N+1)} \otimes A_{c}\right)
\end{gathered}
$$

The Fourier coefficients of the nonlinear terms are computed using alternating-time-frequency method (AFT) [4], that takes advantage of the fast Fourier transform to compute $E$

$$
Q \stackrel{F F T^{-1}}{\longrightarrow} q(t) \rightarrow e(p(t), q(t), \dot{q}(t)) \stackrel{F F T}{\longrightarrow} E
$$

\subsection{Continuation of periodic solutions}

In order to track a branch of periodic solutions, a predictor-corrector method based on pseudoarclength parametrization is used. Denoting $J_{Q}$ and $J_{\omega}$ the Jacobian matrices with respect to $Q$ and $\omega$, respectively, the tangent vector $t_{(i)}$ at a point $\left(Q_{(i-1)}, \omega_{(i-1)}\right)$ along the branch reads

$$
\left[\begin{array}{cc}
J_{Q} & J_{\omega} \\
t_{(i-1)}^{T}
\end{array}\right] t_{(i)}=\left[\begin{array}{l}
0 \\
1
\end{array}\right]
$$

The last equation from (24) prevents the continuation procedure from turning back. The tangent vector is used to compute a prediction for the next step $\left(Q_{(i)}^{(0)}, \omega_{(i)}^{(0)}\right)$ using the step length $\delta_{s}$. Generally, the predicted value does not satisfy Eq. (21). Therefore a correction stage based on Newton's method is performed. In order to be able to deal with turning points, we seek corrections in a direction orthogonal to the tangent vector

$$
\left[\begin{array}{cc}
J_{Q} & J_{\omega} \\
t_{(i)}^{T}
\end{array}\right]\left[\begin{array}{c}
\Delta Q^{(k+1)} \\
\Delta \omega^{(k+1)}
\end{array}\right]=\left[\begin{array}{c}
-h\left(Q_{(i)}^{(k)}, \omega_{(i)}^{(k)}\right) \\
0
\end{array}\right]
$$

with $Q_{(i)}^{(k+1)}=Q_{(i)}^{(k)}+\Delta Q^{(k+1)}, \omega_{(i)}^{(k+1)}=\omega_{(i)}^{(k)}+\Delta \omega^{(k+1)}$. Corrections are performed until the convergence criterion is satisfied.

\section{Numerical example}

In this section, a numerical application of the method based on synthetic data is presented. The studied system consists of two coupled Duffing oscillators. The corresponding equations of motion are given by

$$
\begin{aligned}
& \ddot{q}_{1}(t)+c \dot{q}_{1}(t)+k q_{1}(t)+\mu_{1} q_{1}(t)^{3}+d\left(q_{1}(t)-q_{2}(t)\right)=p(t) \\
& \ddot{q}_{2}(t)+c \dot{q}_{2}(t)+k q_{2}(t)+\mu_{2} q_{2}(t)^{3}+d\left(q_{2}(t)-q_{1}(t)\right)=0
\end{aligned}
$$

From Eq. (26), the nonlinearities are defined by the nonlinear coefficients $\mu_{i}, g_{i}=q_{i}(t)^{3}(i=1,2)$, $b_{1}=[1,0]^{T}$ and $b_{2}=[0,1]^{T}$. Therefore, the extended input vector reads $e(t)=\left[p(t), 0, q_{1}(t)^{3}, q_{2}(t)^{3}\right]^{T}$. The first mass is excited by a single band-limited $(0-80 \mathrm{rad} / \mathrm{s})$ normally distributed random signal (5000 points) repeated 8 times. Its root-mean-square (r.m.s.) value is equal to $3 N$. Numerical integration of the equations of motion was performed using fourth order Runge-Kutta method. The last 5000 points of the result of the numerical integration, resampled at $200 \mathrm{rad} / \mathrm{s}$, are used as input to generate the extended input vector. Remark that the input band was chosen to encompass the third harmonic of the highest natural frequency. The model order $n=4$ is determined from the inspection of the singular value plot depicted in Fig. 2 with $i=40$ block rows. A jump of four orders of magnitude between model order four and five is observed. Also, as shown in Fig. 2, an excellent agreement between the theoretical and identified FRF of the underlying linear system is observed.

Fig. 3 displays the complex and frequency-dependent estimation of the nonlinear coefficients determined from the extended FRF [9]. Note that this step is unnecessary for the continuation procedure and only serves as an indicator of the quality of the identification. Effectively, a correctly estimated system should lead to an almost constant nonlinear coefficient over the frequency range of interest and an imaginary part several orders of magnitude below the real part. In this case, a 

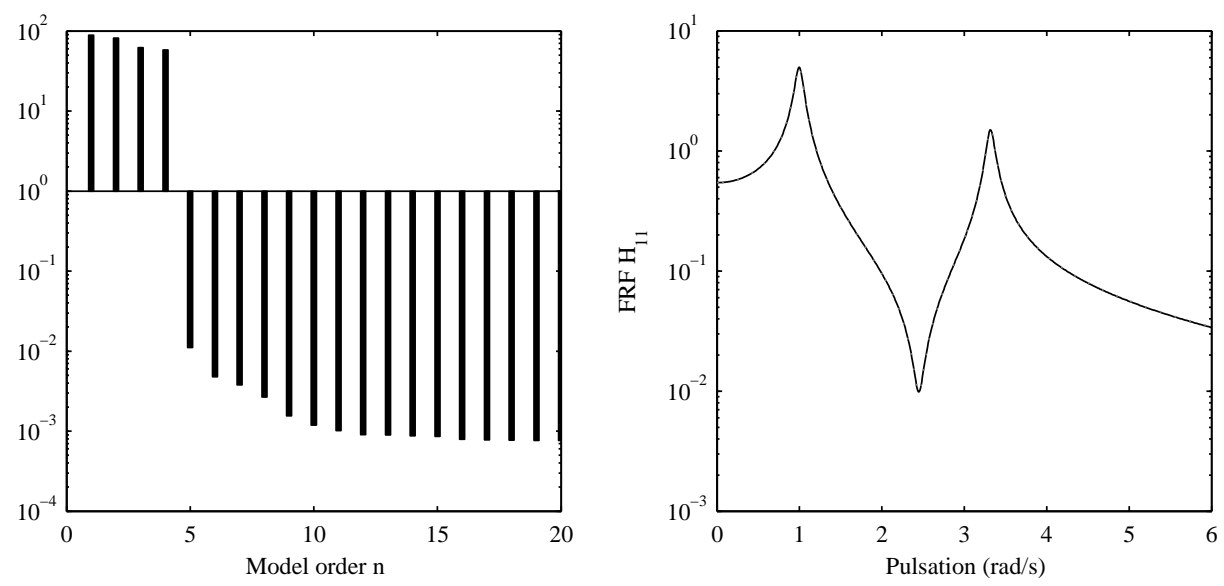

Figure 2: Left : plot of the twenty first singular values with $i=30$. Right : theoretical (grey dotted line) and identified (black solid line) FRF of the underlying linear system.
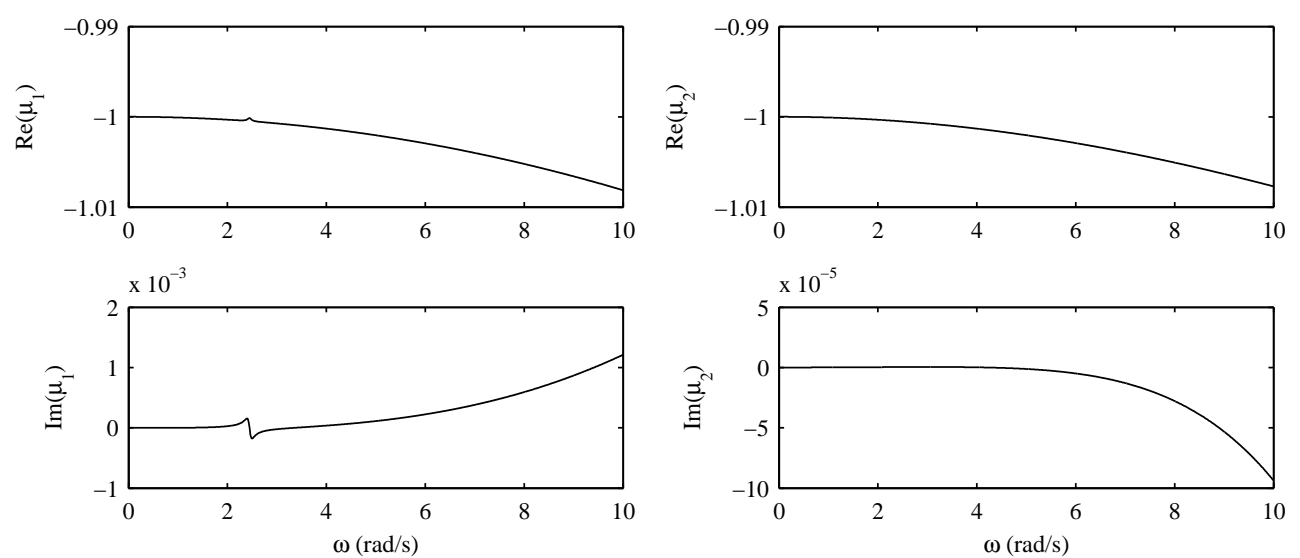

Figure 3: Real and imaginary parts of the estimated nonlinear coefficients $\mu_{1}$ and $\mu_{2}$.

variation of less than $1 \%$ of the real part of the nonlinear coefficients in the frequency range of interest is observed. The imaginary parts are three and four orders of magnitude lower that the real parts for the first and second nonlinear coefficient, respectively.

The identified $B_{c}$ matrix in the physical state-space domain, denoted $\tilde{B}_{c}$, computed using Eq. $(10,13)$ yields

$$
\tilde{B}_{c}=\left[\begin{array}{cccc}
0.016 & 0 & 0.016 & 0 \\
0 & 0 & 0 & 0.016 \\
0.997 & 0 & 0.998 & 0 \\
0 & 0 & 0 & 0.998
\end{array}\right]
$$

The values of the nonlinear coefficients are close to the original model in Eq. (4). However, the structure of $\tilde{B}_{c}$ is not conform with the theoretical expectation, since undesired terms that are approximatively two orders of magnitude smaller than the nonlinear and the forcing coefficients are present in the first block row of $\tilde{B}_{c}$. The effect of these undesired terms can be apprehended by rewriting the identified physical state-space model into the physical space. The identified system reads

$$
\left[\begin{array}{c}
\dot{x}_{1} \\
\dot{x}_{2} \\
\dot{x}_{3} \\
\dot{x}_{4}
\end{array}\right]=\left[\begin{array}{cccc}
0 & 0 & 1 & 0 \\
0 & 0 & 0 & 1 \\
-\tilde{k}-\tilde{d} & \tilde{d} & -\tilde{c} & 0 \\
\tilde{d} & -\tilde{k}-\tilde{d} & 0 & -\tilde{c}
\end{array}\right]\left[\begin{array}{c}
x_{1} \\
x_{2} \\
x_{3} \\
x_{4}
\end{array}\right]+\left[\begin{array}{cccc}
B_{1} & 0 & B_{2} & 0 \\
0 & 0 & 0 & B_{3} \\
\tilde{\mu}_{f} & 0 & \tilde{\mu}_{1} & 0 \\
0 & 0 & 0 & \tilde{\mu}_{2}
\end{array}\right]\left[\begin{array}{c}
p \\
0 \\
q_{1}^{3} \\
q_{2}^{3}
\end{array}\right]
$$



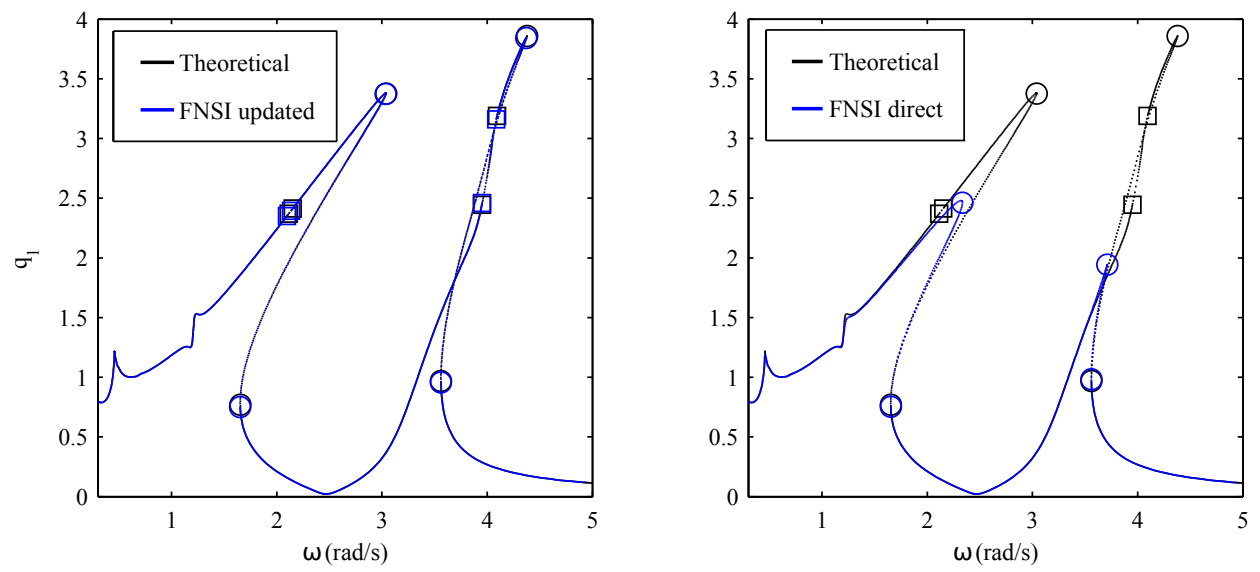

Figure 4: Frequency response curves of the theoretical (black) and identified model (blue). Left: updated FNSI model. Right: original FNSI model.

where the tildes denote the coefficients identified using the FNSI method and expressed in the physical state-space domain. The terms $B_{i}(i=1 . .3)$ denote the undesired terms of the $\tilde{B}_{c}$ matrix. Using the matrix $C$ (which is the exact due to (11)), the reconstructed model in the physical-domain is written by

$$
\begin{aligned}
& \ddot{q}_{1}+\tilde{c} \dot{q}_{1}+\tilde{k} q_{1}+\tilde{\mu}_{1} q_{1}^{3}+\tilde{d}\left(q_{1}-q_{2}\right)-3 B_{2} q_{1}^{2} \dot{q}_{1}=\tilde{\mu}_{f} p(t)+B_{1} \dot{p}(t) \\
& \ddot{q}_{2}+\tilde{c} \dot{q}_{2}+\tilde{k} q_{2}+\tilde{\mu}_{2} q_{2}^{3}+\tilde{d}\left(q_{2}-q_{1}\right)-3 B_{3} q_{2}^{2} \dot{q}_{2}=0
\end{aligned}
$$

It is seen that the term $B_{1}$ modify the excitation while the terms $B_{2}$ and $B_{3}$ constitute spurious nonlinearities corresponding to Van der Pol damping added to the identified model. These terms are conform with the assumed nonlinear basis functions and must be forced to zero in order to recover a coherent identified system.

The result of the numerical continuation is depicted in Fig. 4 for a forcing amplitude of $2 N$. Solid and dotted lines correspond to stable and unstable periodic responses, respectively. Black and blue lines correspond to the theoretical and identified frequency response curves of the system, respectively. The circles and the squares represent fold and Neimark-Sacker bifurcations, respectively. Both responses of the theoretical and identified model were obtained using the continuation procedure. The frequency response curve of the identified model without and with the undesired terms $B_{i}$ are depicted in the left and the right graph, respectively.

The frequency response curve of the identified model when the spurious terms (termed FNSI updated) have been removed matches almost exactly the theoretical predictions. Both fold and NeimarkSacker bifurcations are identified in agreement with the theoretical prediction. On the contrary, as observed on the right plot, even if these spurious terms are small compared to the identified nonlinear coefficients, they lead to strongly erroneous results and therefore must be removed from $\tilde{B}_{c}$.

\section{Conclusion}

In this paper, an algorithm coupling the frequency nonlinear subspace identification method and a harmonic balance based continuation method for the simulation has been presented. The objective of this procedure is to obtain the nonlinear frequency response curves of a nonlinear system directly from random measurements. Since the identification procedure is carried out in the state space domain, the simulation is also performed in the state space domain, which prevents from reconstructing the identified model in physical space. An expression of the similarity transformation matrix which allows the identification of spurious nonlinearity has been presented. The method has been tested on a numerical application with a two degree of freedom Duffing oscillator. The theoretical and identified frequency response curves are in good agreement. The nature and location of the bifurcations is also recovered. The origin of the spurious terms in the identified matrix $B_{c}$ has not been yet identified, which will be the object of further work. 


\section{References}

[1] DE Adams and RJ Allemang. A new derivation of the frequency response function matrix for vibrating non-linear systems. Journal of Sound and Vibration, 227(5):1083-1108, 1999.

[2] EL Allgower and K Georg. Numerical continuation methods: an introduction, volume 13. Springer Science \& Business Media, 2012.

[3] F Barillon, J-J Sinou, J-M Duffal, and L Jézéquel. Non-linear dynamics of a whole vehicle finite element model using a harmonic balance method. International Journal of Vehicle Design, 63(4):387-403, 2013

[4] TM Cameron and JH Griffin. An alternating frequency/time domain method for calculating the steady-state response of nonlinear dynamic systems. Journal of applied mechanics, 56(1):149$154,1989$.

[5] T Detroux, L Renson, and G Kerschen. The harmonic balance method for advanced analysis and design of nonlinear mechanical systems. In Nonlinear Dynamics, Volume 2, pages 19-34. Springer, 2014.

[6] DJ Ewins, B Weekes, and A delli Carri. Modal testing for model validation of structures with discrete nonlinearities. Phil. Trans. R. Soc. A, 373(2051):20140410, 2015.

[7] L Garibaldi, S Marchesiello, and E Bonisoli. Identification and up-dating over the z24 benchmark. Mechanical Systems and Signal Processing, 17(1):153-161, 2003.

[8] G Kerschen, K Worden, AF Vakakis, and J-C Golinval. Past, present and future of nonlinear system identification in structural dynamics. Mechanical systems and signal processing, 20(3):505-592, 2006.

[9] S Marchesiello and L Garibaldi. A time domain approach for identifying nonlinear vibrating structures by subspace methods. Mechanical Systems and Signal Processing, 22(1):81-101, 2008.

[10] T McKelvey, H Akçay, and L Ljung. Subspace-based multivariable system identification from frequency response data. Automatic Control, IEEE Transactions on, 41(7):960-979, 1996.

[11] J-P Noël and G Kerschen. Frequency-domain subspace identification for nonlinear mechanical systems. Mechanical Systems and Signal Processing, 40(2):701-717, 2013.

[12] J-P Noël, G Kerschen, E Foltête, and S Cogan. Grey-box identification of a non-linear solar array structure using cubic splines. International Journal of Non-Linear Mechanics, 67:106-119, 2014.

[13] P Van Overschee and BL De Moor. Subspace identification for linear systems: TheoryImplementationApplications. Springer Science \& Business Media, 2012.

[14] G Von Groll and DJ Ewins. The harmonic balance method with arc-length continuation in rotor/stator contact problems. Journal of sound and vibration, 241(2):223-233, 2001. 